\title{
Criminologie
}

\section{Entre les attentes face à la judiciarisation et l'issue des procédures : réflexion à partir d'une étude sur le traitement judiciaire des causes de violence conjugale}

\section{Danielle Laberge et Sonia Gauthier}

Volume 33, numéro 2, automne 2000

Problèmes sociaux et système pénal

URI : https://id.erudit.org/iderudit/004723ar

DOI : https://doi.org/10.7202/004723ar

Aller au sommaire du numéro

Éditeur(s)

Les Presses de l'Université de Montréal

ISSN

0316-0041 (imprimé)

1492-1367 (numérique)

Découvrir la revue

Citer cet article

Laberge, D. \& Gauthier, S. (2000). Entre les attentes face à la judiciarisation et l'issue des procédures : réflexion à partir d'une étude sur le traitement judiciaire des causes de violence conjugale. Criminologie, 33(2), 31-53. https://doi.org/10.7202/004723ar
Résumé de l'article

Dans les années 70, la violence et les autres préjudices et injustices subis par les femmes ont été dénoncés publiquement. Devant l'ampleur du phénomène de la violence conjugale et devant le constat que les tribunaux n'étaient pas souvent saisis de ces affaires et que celles-ci ne se rendaient pas souvent au terme des procédures, divers groupes ont aussi réclamé que les instances pénales agissent avec plus de vigueur dans les situations impliquant de la violence conjugale. Plusieurs politiques furent alors implantées pour accroître la criminalisation des comportements violents entre conjoints. Cet article porte sur le fossé entre les attentes face à la judiciarisation et ce que nous avons observé en ce qui a trait à la proportion de conjoints coupables et aux peines qui leur ont été infligées. Nous traitons d'abord des problèmes associés à la judiciarisation, puis de la double victimisation, du faible taux de culpabilité et, finalement, de la peine infligée aux agresseurs. 


\title{
Entre les attentes face à la judiciarisation et l'issue des procédures : réflexion à partir d'une étude sur le traitement judiciaire des causes de violence conjugale
}

\author{
Sonia Gauthier ${ }^{1}$ \\ Chercheure post-doctorale \\ Centre de recherche interdisciplinaire \\ sur la violence familiale et la violence \\ faite aux femmes (CRI-VIFF) \\ Université de Montréal \\ gauthso@magellan.umontreal.ca \\ Danielle Laberge \\ Professeure \\ Département de sociologie \\ Université du Québec à Montréal \\ laberge.danielle@uqam.ca
}

RÉSUMÉ - Dans les années 70, la violence et les autres préjudices et injustices subis par les femmes ont été dénoncés publiquement. Devant l'ampleur du phénomène de la violence conjugale et devant le constat que les tribunaux n'étaient pas souvent saisis de ces affaires et que celles-ci ne se rendaient pas souvent au terme des procédures, divers groupes ont aussi réclamé que les instances pénales agissent avec plus de vigueur dans les situations impliquant de la violence conjugale. Plusieurs politiques furent alors implantées pour accroître la criminalisation des comportements violents entre conjoints. Cet article porte sur le fossé entre les attentes face à la judiciarisation et ce que nous avons observé en ce qui a trait à la proportion de conjoints coupables et aux peines qui leur ont été infligées. Nous traitons d'abord des problèmes associés à la judiciarisation, puis de la double victimisation, du faible taux de culpabilité et, finalement, de la peine infligée aux agresseurs.

1. L'article est tiré en partie de la thèse de doctorat de l'auteure, qui a bénéficié d'une bourse de doctorat du CQRS.

Criminologie, vol. 33, $n^{\circ} 2$ (2000) 
ABSTRACT - During the seventies, violence and other prejudices and injustices to which women were subjected were publicly denounced. Because of the extent of domestic violence and infrequent and inconsistent recourse to the criminal justice system, pressure groups also demanded more systematic prosecution of violence against women, especially violence within a domestic context. Many public policies were developed and implemented to attain this goal. Our article will examine the gap between expectations regarding the use of the penal system and the actual treatment reserved for spouses found guilty. We will first discuss problems associated with the use of criminalization; we will then focus on double victimization, low rate of conviction, and finally the sentences imposed.

Aujourd'hui, la judiciarisation accrue des conjoints violents fait suite à un fort mouvement de pression amorcé par les groupes de femmes et repris par le mouvement féministe (Beaudry, 1984; Davis, 1988; Buzawa et Buzawa, 1990; Lavergne, 1997). La violence conjugale, d'abord perçue comme un problème social, fut ensuite rapidement considérée comme un crime (Walker, 1990), ce qui a conduit à d'importantes transformations quant aux solutions réclamées et mises en œuvre pour lutter contre ce phénomène. Les revendications des groupes de pression étaient doubles. D'une part, que les comportements violents entre conjoints soient interprétés comme des crimes, au même titre que le seraient ceux qui impliquent des personnes ayant d'autres liens, ou des étrangers (MacLeod, 1987; British Columbia Task Force on Family Violence, 1992). D'autre part, que les intervenants pénaux traitent ces actes au même titre que les autres, voire plus durement (Davis, 1988). Pour Soler (1987), il en allait de la crédibilité même de l'appareil pénal.

La première moitié des années 1980 a vu proliférer en Amérique du Nord diverses politiques d'intervention mises en place pour favoriser la criminalisation de la violence conjugale. Signalons d'abord l'adoption par la Chambre des communes du Canada, au début des années 1980, d'une motion incitant les policiers à porter eux-mêmes les accusations contre l'agresseur dans les événements impliquant des conjoints. Quant au Québec, le ministère de la Justice et le Solliciteur général ${ }^{2}$ ont proposé une Politique d'intervention en matière de violence conjugale, publiée en 1986. Cette politique, en vigueur au moment de notre recherche, a été revue en $1995^{3}$. Au plan local, le Service de police de la Commu-

2. Aujourd'hui, ministère de la Sécurité publique

3. La politique de 1995, engageant le gouvernement du Québec dans une action concertée, réaffirme les principes de la politique de 1986 et implique quatre ministères (ministères de la Santé et des Services sociaux, de la Justice, de la Sécurité publique et de l'Éducation) et deux secrétariats (secrétariats à la Condition féminine et à la Famille). 
nauté urbaine de Montréal (SPCUM) a développé une politique d'intervention, soit la Procédure spécifique 5059 - Violence conjugale, implantée le $1^{\text {er }}$ septembre 1986 et modifiée plusieurs fois depuis. Mentionnons finalement l'entrée en vigueur, en septembre 1996, d'une nouvelle disposition du code criminel concernant les principes de la détermination de la peine. On y déclare entre autres que les mauvais traitements perpétrés contre son conjoint ou ses enfants doivent être considérés comme une circonstance aggravante, ce dont le juge doit tenir compte lorsqu'il décide de la peine à infliger (art. 718.2).

Les attentes face à la répression du phénomène de violence conjugale ont été importantes et elles ralliaient des acteurs sociaux très divers. Ces attentes se fondaient pour une large part sur une participation active et importante du système de justice pénale. Pour plusieurs, les actions qui s'en sont suivies n'ont pas été suffisantes : trop peu de cas poursuivis, trop de poursuites abandonnées, sentences trop clémentes. À quoi ces situations sont-elles attribuables ? Comment accroître la performance du système ? Cet accroissement de la performance est-il souhaitable ? Les questions ainsi soulevées, dont nous n'avons donné ici que quelques exemples, sont à la fois nombreuses et complexes. Dans cet article, nous souhaitons contribuer à la réflexion sur l'usage du système pénal comme réponse spécifique à des affaires de violence conjugale. Notre réflexion se fonde sur les résultats d'une recherche empirique menée à Montréal en 1992-1993.

Nous examinerons d'abord les attentes face à la judiciarisation. Nous ferons ensuite état des problèmes soulevés par l'application de la loi et par le recours à la judiciarisation en tant que réponse à la violence conjugale. Nous enchaînerons sur la double victimisation. Cette introduction servira de trame de fond pour discuter de l'issue des causes de violence conjugale et de la peine infligée aux coupables. Le fonctionnement de la justice pénale est régi par des règles, des contraintes et des procédures qui lui sont propres. Leur prise en compte est nécessaire pour comprendre les décisions rendues.

\section{Les attentes face à la judiciarisation}

La capacité de l'appareil pénal de traiter des causes impliquant de la violence conjugale et la pertinence de ce type d'intervention ne sont plus un discours provenant des seuls groupes militants. Elle est réaffirmée de toutes parts (Québec, 1986, 1995; Gondolf et McFerron; 1989; Office 
des Nations Unies (ONU), 1989; Canada, 1991; Côté, 1991; Morier et al., 1991; Busch et Robertson, 1993). Les arguments en faveur de la judiciarisation se rattachent globalement à deux des finalités attribuées à ce type d'intervention : la prévention générale et la prévention spéciale.

La prévention générale concerne la capacité de l'appareil pénal d'influencer le comportement des membres de la société. La judiciarisation des affaires de violence conjugale est perçue comme étant un moyen d'affirmer le caractère inacceptable et répréhensible de ces actes. On s'attend à ce que cette pratique, notamment l'imposition de sentences exemplaires, ait un effet de dissuasion générale et participe ainsi à la diminution de l'incidence de la violence conjugale (Côté, 1991). La seconde finalité, la prévention spécifique, vise les individus déjà ciblés comme étant violents. On prête à la justice pénale et particulièrement à la détention une efficacité réelle, non seulement comme moyen de protection immédiate des victimes, mais également pour mettre fin à la violence à court et à long termes. La crainte de la récidive pour justifier l'intervention pénale est alimentée par un argument fort répandu : les personnes qui ont eu des comportements très violents à l'égard de leur conjointe ou qui l'ont assassinée ont antérieurement posé des gestes moins graves. Il y aurait donc escalade de la violence. Par conséquent, il faut intervenir dès les premières manifestations ou intentions de violence pour prévenir une récidive (Burris et Jaffe, 1983; Martin, 1987; ONU, 1989; Buzawa et Buzawa, 1990; Statistique Canada, 1994; Law Reform Commission of Nova Scotia, 1995). Pour les partisans de la judiciarisation, l'intervention pénale est très souvent perçue comme un des moyens les plus efficaces pour freiner cette escalade ou faire cesser la violence, sinon le seul (ONU, 1989).

\section{Des problèmes liés à la judiciarisation}

Malgré ce consensus, la judiciarisation des événements de violence conjugale a fait l'objet de sérieuses critiques. Plusieurs auteurs ont noté des problèmes dans la manière dont les intervenants pénaux appliquent la loi : les policiers ne portent pas assez d'accusations; certains demandent encore aux femmes si elles veulent porter plainte, alors que ce sont eux qui doivent le faire; les accusés sont trop facilement remis en liberté; les procédures sont trop longues; les accusés ne sont pas souvent trouvés coupables; les sentences sont trop légères; etc. Ces problèmes se traduiraient, en appa- 
rence, par un traitement plus clément des conjoints accusés comparativement aux accusés qui s'en prennent à des inconnus (Soler, 1987; ONU, 1989; Côté, 1991; Busch et al., 1992; Law Reform Commission of Nova Scotia, 1995) ${ }^{4}$. Ces problèmes seraient imputables à l'attitude des intervenants pénaux (policiers, procureurs de la couronne et juges) et des gouvernements envers la violence conjugale. Certains mettent l'accent sur le manque de volonté des gouvernements d'apporter des solutions efficaces pour régler le problème de la violence conjugale et le manque d'intérêt de la part des agents pénaux face à la protection des femmes battues (Law Reform Commission of Nova Scotia, 1995). D'autres déplorent que les acteurs pénaux ne considèrent et ne traitent pas la violence conjugale comme un problème extrêmement sérieux (Côté, 1991; Busch et al., 1992); qu'ils se représentent ces événements comme des disputes familiales, des affaires privées dans lesquelles il vaut mieux ne pas intervenir (Soler, 1987; Buzawa et Buzawa, 1990; Morier et al., 1991); qu'ils croient qu'une intervention pénale risque de mettre en péril l'unité familiale (Davis, 1988; ONU, 1989); que les femmes sont traitées de façon sexiste au sein de l'appareil pénal (Brown, 1991); que les intervenants et les décideurs ne reconnaissent pas que la violence conjugale n'est pas une agression entre deux personnes, mais bien l'agression d'un homme contre une femme, un crime spécifiquement genré (gender-based crime), qui devrait être reconnu comme tel dans le code criminel (Law Reform Commission of Nova Scotia, 1995).

Par ailleurs, des auteurs ont insisté sur les problèmes qui concernent le recours même au système de justice pénale pour répondre à la violence conjugale et, plus largement, aux conflits interpersonnels. Il s'agit, entre autres, des coûts individuels et sociaux de l'intervention pénale (Brodeur et Landreville, 1979; Landreville et al., 1981; ASRSQ, 1989; Ford et Regoli, 1993) et des problèmes découlant de la manière dont fonctionne cet appareil. À ce sujet, soulignons premièrement le fait que ce soit un système contradictoire (adversarial) (Jones, 1994). Deuxièmement, dans un litige de nature criminelle, la victime n'est pas une personne en particulier, mais l'État. La personne agressée est un témoin dans ces causes qui opposent l'État, représenté par le procureur de la couronne, à celui accusé d'avoir transgressé les règles. Par conséquent, le dépôt d'une plainte ou son retrait n'est pas conditionnel à la volonté de l'agressée, en théorie du

4. Cette opinion ne fait cependant pas l'unanimité. Voir à ce sujet MacLeod et Picard (1989); Demers (1990); Morier et al. (1991). 
moins, mais relève de la décision du procureur de la couronne. Il se peut alors que les désirs ou les besoins des victimes, traduits par exemple par une demande d'arrêt des procédures ou un refus de témoigner, soient subordonnés aux intérêts des intervenants judiciaires : rendre justice, démontrer que l'on prend ces situations en charge. Troisièmement, la victime s'expose au contre-interrogatoire mené par l'avocat de la défense, expérience qui peut être très éprouvante. Finalement, bon nombre de poursuites criminelles en violence conjugale sont abandonnées pendant les procédures. Cette situation, en partie imputable au refus des victimes de témoigner lors de l'enquête préliminaire ou pendant le procès, irrite les intervenants pénaux (Cretney et Davis, 1997; McGuire, 1997; Rondeau et al., 1998). Ils ont l'impression de perdre leur temps avec des victimes qui refusent d'être aidées (Ford, 1991).

Le recours à la judiciarisation de la violence conjugale s'inscrit dans une double tendance plus générale : la conception de la criminalisation comme solution pertinente et nécessaire dans la gestion de certains problèmes sociaux et la présence croissante de l'État dans la sphère privée. Cette double tendance produit également des effets délétères. Dans le premier cas, signalons : a) la recrudescence des demandes du public pour une application plus ferme des lois pénales, un contrôle et une répression plus sévères de la criminalité (Gelles et Straus, 1988; Laberge et Landreville, 1994); b) l'effet de diversion, qui consiste à « [...] laisser croire que l'incarcération est le principal, sinon le seul moyen efficace pour solutionner un problème. Une législation très punitive ou l'incarcération de quelques boucs émissaires donnent l'impression que l'on fait tout pour solutionner le problème, elles satisfont l'opinion publique et évitent d'apporter des solutions plus complexes et plus coûteuses » (Laberge et Landreville, 1994 : 1078); c) le caractère discriminatoire de cette intervention contre les personnes défavorisées socialement (Brodeur et Landreville, 1979; Landreville et al., 1981; Davis, 1988); d) les attentes irréalistes face à cet appareil de contrôle, qui devrait être utilisé avec modération (Commission de réforme du droit du Canada, 1976; MacLeod et Picard, 1989). Dans le second cas, mentionnons : a) le paradoxe contenu dans le recours aux institutions étatiques pour soutenir et protéger les femmes, alors qu'on accuse ces mêmes institutions de participer à la reproduction des inégalités (Morgan, 1981); b) le fait qu'une des caractéristiques de l'intervention pénale est de réduire le problème à une question de responsabilité individuelle, ce qui évacue le caractère structurel de la violence exercée contre les femmes. 


\section{La double victimisation}

Plusieurs de ceux qui relèvent des problèmes dans l'application de la loi ou qui s'inquiètent des problèmes associés à l'intervention pénale se rejoignent autour d'une même préoccupation : l'intervention pénale risque de victimiser doublement les femmes aux prises avec la violence exercée par leur conjoint. Outre certaines situations déplorables, comme lorsque la victime se retrouve menacée d'outrage au tribunal pour un refus de témoigner contre son conjoint, la double victimisation se manifeste sous plusieurs formes. Si certains considèrent qu'une poursuite criminelle représente le seul moyen pour certaines victimes d'acquérir du contrôle et du pouvoir, d'autres n'y voient que le remplacement d'un contrôle par un autre (Davis, 1988); l'intervention pénale est perçue comme une forme de domination dépossédant encore plus les victimes de leur pouvoir (MacLeod, 1989); elle ne répond pas à leurs attentes ou néglige leurs besoins de sécurité et de justice (Brown, 1991; Law Reform Commission of Nova Scotia, 1995; Québec, 1995); les intervenants pénaux prennent des décisions qui vont à l'encontre des intérêts et des volontés des victimes (Davis, 1988; MacLeod et Picard, 1989; Buzawa et Buzawa, 1990). La conséquence la plus grave de cette double victimisation - et ce, peu importe à quel problème on l'attribue et de quelle manière elle se manifeste - est que les victimes peuvent hésiter à recourir de nouveau ou pour une première fois à l'intervention judiciaire, même si elles ont besoin d'aide et de protection. Il est d'ailleurs connu que les victimes peuvent subir plusieurs agressions avant de demander de l'aide (MacLeod, 1987; Morier et al., 1991).

Pour plusieurs, le moyen d'éviter cette double victimisation réside dans un changement profond des mentalités des acteurs pénaux, dans un meilleur soutien aux victimes pendant les procédures, dans des réajustements de la pratique pénale. Autrement dit, régler de l'intérieur les problèmes de mise en œuvre et la source de ces problèmes. À l'extrême, on créera des tribunaux spécialisés dans le traitement de ce type d'affaire, comme on l'a fait à Winnipeg (Manitoba) en 1990. Le tribunal de la violence familiale a été créé expressément dans le but de traiter ce genre de cause avec la même vigueur que celles impliquant d'autres parties. Les procureurs de la couronne et les juges recrutés pour œuvrer dans ce tribunal ont été spécialement formés aux spécificités de l'intervention judiciaire impliquant des personnes - partenaires, personnes âgées et enfants - qui ont « [...] un lien de confiance, de dépendance ou de parenté avec l'accusé » (Ursel, 1994 : 4). Pour d'autres, qui peuvent 
également souligner l'importance d'améliorer l'intervention des acteurs pénaux et qui admettent que la judiciarisation peut être incontournable dans certains cas, la recherche d'alternatives à la judiciarisation s'avère nécessaire vu les divers problèmes associés au recours à l'appareil pénal pour traiter ce genre de situation. Dans le contexte actuel, cependant, il semble qu'il y ait bien peu d'ouvertures pour ce genre de réorientation.

\section{La démarche de recherche}

Les données dont nous traiterons dans cet article ont été recueillies dans le cadre d'une recherche sur la détention sous garde et la mise en liberté provisoire, menée à la cour du Québec de Montréal, chambre criminelle et pénale (Amoretti et al., 1996). Les prévenus ayant comparu détenus pour une nouvelle plainte, pendant l'année administrative 1992-1993, ont constitué la population de base pour la recherche sur la détention sous garde et la mise en liberté provisoire. Nous avons procédé par échantillonnage systématique et avons retenu un dossier sur 2,5, ce qui a constitué un échantillon composé de 2079 dossiers ouverts entre le 16 novembre 1992 et la fin du mois d'avril 1993. Sur les 2079 dossiers ouverts, nous avons identifié 262 dossiers impliquant des hommes accusés dans une affaire de violence conjugale.

Notre article porte sur ces 262 causes. Nous avons sélectionné comme étant de la violence conjugale les événements qui impliquent un homme accusé d'une infraction contre la personne, et dont la conjointe (de droit, de fait ou amie intime) ou ex-conjointe est la victime présumée et ce, qu'ils cohabitent ou non. À l'époque où nous avons mené la recherche, la majorité des conjoints comparaissaient détenus (Gravel, 1993) ${ }^{5}$. On peut donc

présumer que les dossiers que nous avons retenus représentent la majorité des dossiers d'hommes qui ont comparu pour une nouvelle plainte dans une affaire de violence conjugale à la cour du Québec, pendant la période couverte par notre recherche ${ }^{6}$. Ces causes ont été comparées à 369 événe-

5. Ce n'est plus le cas aujourd'hui. En 1994, soit après la période de cueillette de nos données, le code criminel a conféré aux agents de la paix et aux fonctionnaires responsables le pouvoir d'imposer certaines conditions aux suspects lorsqu'ils les remettent en liberté. La liste des conditions a été allongée une première fois en 1997 et une seconde fois en 1999.

6. Les causes de meurtres sont entendues à la cour supérieure. C'est pourquoi notre échantillon ne comporte aucune accusation de ce type. Par ailleurs, nous avons retiré de la présente analyse les cas où le conjoint a été accusé d'une infraction contre les biens de sa conjointe ou d'une omission de se conformer à une ordonnance du tribunal. 
ments concernant des hommes accusés d'une infraction contre la personne, mais dans un contexte autre que familial ${ }^{7}$.

Les données sur l'issue des procédures judiciaires dans chacune des causes étudiées proviennent de la banque de données du ministère de la Justice du Québec. Les informations obtenues nous permettent de dresser un portrait général de la cause, de son enclenchement jusqu'à la décision rendue. Par contre, leur caractère demeure limité à plusieurs égards. C'est ainsi que nous ne connaissons pas les justifications des diverses décisions. Par ailleurs, ce type de cueillette de données ne permet pas de retracer les événements subséquents qui peuvent être en lien avec la cause en question. À titre d'exemple, nous ne savons pas si, dans les causes où un arrêt des procédures a été ordonné, le justiciable a fait l'objet d'une reprise des poursuites. D'autres recherches seront nécessaires pour offrir un portrait plus complet de la situation.

\section{Résultats et éléments de réflexion}

L'issue des causes

Lorsque nous avons reçu les informations sur l'issue des procédures judiciaires, $89,3 \%(\mathrm{~N}=234)$ des dossiers des conjoints étaient clos, i.e. qu'une décision avait été prise par le tribunal. Le tiers des conjoints a été finalement accusé d'une infraction grave contre la personne ${ }^{8}$, mais la majorité a fait face à une accusation pour un autre type d'infraction contre la personne $e^{9}(67,5 \%, N=158)^{10}$. La politique enjoignant les policiers à porter systématiquement eux-mêmes des accusations dans les affaires de violence conjugale a contribué à accroître de façon substantielle le nombre de ces situations que doivent gérer les tribunaux. Si les policiers renvoient ces affaires au tribunal, ce n'est pas parce qu'ils

7. À la différence des conjoints, les autres hommes ne comparaissaient pas tous détenus. Il faut donc garder à l'esprit que les données que nous présentons quant à l'issue des causes et aux peines infligées à ces autres hommes ne sont généralisables qu'à la cohorte des hommes qui ont comparu détenus. Les résultats seraient peut-être différents si nous avions fait les analyses sur tous les accusés, qu'ils aient comparu libres ou détenus.

8. Agression armée ou infliction de lésions corporelles, voies de fait grave, enlèvement, séquestration, tentative de meurtre.

9. Menaces, intimidation, voies de fait qui ne causent pas de blessures, agression sexuelle autre que grave ou armée.

10. Pour les hommes accusés dans un autre type d'événement, ces proportions sont diamétralement opposées : 63,4\% ( $\mathrm{N}=234)$ ont été accusés d'une infraction grave contre la personne. 
jugent qu'il n'y a pas d'autres issues ou d'autres modes de résolution de problème, par exemple, mais bien parce qu' ils sont tenus de le faire dans bon nombre de situations. En effet, presque une décennie après l'adoption de la Politique d'intervention en matière de violence conjugale, on évalue que : «La mise en vigueur de cette politique [la politique de 1986] a entraîné une augmentation importante du nombre de cas signalés à la police. De plus, les pratiques d'intervention policière et judiciaire se sont modifiées, amenant une judiciarisation presque systématique des cas signalés à la police » (Québec, 1995 : 57). C'est peut-être également ce qui explique l'importante proportion d'accusations d'agression mineure contre la personne. Ce genre d'événement, dans un autre contexte que la violence conjugale, aurait peut-être fait l'objet de "diversion » (aucune accusation portée; résolution de conflit sur les lieux de l'événement; renvoi vers des ressources appropriées).

L'examen de l'issue des causes nous apprend d'abord que $26,5 \%$ des conjoints ont plaidé coupable (tableau 1). Diverses raisons peuvent expliquer la décision de plaider coupable. Par exemple, les accusés veulent en finir avec les procédures judiciaires, ils considèrent que la preuve contre eux est forte ou alors ils se savent coupables et l'admettent (Ericson et Baranek, 1982). Le plaidoyer de culpabilité est l'issue qui distingue le plus les conjoints des hommes accusés dans un autre type de situation : ces derniers plaident majoritairement coupables (62,6\%). Nous observons également que le juge a déclaré coupable $5,1 \%$ des conjoints. La faible proportion de déclaration de culpabilité n'est pas exclusive aux causes de violence conjugale puisque pour les autres hommes, le même phénomène se constate $(6,0 \%)$.

Ainsi, parmi les conjoints accusés, $31,6 \%$ sont coupables au terme des procédures. Cependant, les causes se sont terminées par une autre issue que la culpabilité dans $68,4 \%$ des cas. La décision la plus courante est une libération des accusations $(39,7 \%)$. Cette décision ne peut être prise que dans le contexte d'une enquête préliminaire. Rappelons que cette dernière procédure n'est pas mise en œuvre dans tous les dossiers. La libération survient lorsque le juge de paix estime que la preuve contre l'accusé est insuffisante pour qu'il subisse son procès (art. 548 C.cr.). Une des raisons expliquant cette issue est l'absence de témoins pour faire la preuve du délit. Par ailleurs, au stade du procès, le juge a acquitté $12,8 \%$ des conjoints, mettant ainsi un terme à la poursuite intentée contre ces justiciables. Finalement, un arrêt des procédures fut prononcé dans 10,7 \% des cas. Le type d'arrêt des procédures dont il s'agit vraisemblablement ici est 
T A B LEA U 1

L'issue des procédures

\begin{tabular}{|l|cc|cc|}
\hline & \multicolumn{2}{|c|}{ Violence conjugale } & \multicolumn{2}{c|}{$\begin{array}{c}\text { Autres infractions } \\
\text { contre la personne }\end{array}$} \\
\hline Issue ${ }^{1}$ & $\mathbf{N}$ & $\%$ & $\mathbf{N}$ & $\%$ \\
$\begin{array}{l}\text { culpabilité } \\
\quad \text { plaide coupable }\end{array}$ & 62 & 26,5 & 231 & 62,6 \\
déclaré coupable & 12 & 5,1 & 22 & 6,0 \\
$\quad$ Total culpabilité) & $(74)$ & $(31,6)$ & $(253)$ & $(68,6)$ \\
autres issues & & & & \\
libéré des accusations & 93 & 39,7 & 80 & 21,7 \\
acquitté & 30 & 12,8 & 16 & 4,3 \\
arrêt des procédures & 25 & 10,7 & 12 & 3,2 \\
accusation retirée & 12 & 5,1 & 8 & 2,2 \\
$\quad$ Total autres issues) & $(160)$ & $(68,4)$ & $(116)$ & $(31,4)$ \\
Total & $\mathbf{2 3 4}$ & $\mathbf{1 0 0}$ & $\mathbf{3 6 9}$ & $\mathbf{1 0 0}$ \\
\hline
\end{tabular}

${ }^{1} \mathrm{Khi}^{2}:$ 85,06; p. : 0,00; V de Cramer : 0,38.

celui qui est ordonné par le procureur général (art. 579 C.cr.). À ce sujet, il faut noter que les procédures peuvent être reprises et ce, sans qu'il soit nécessaire de déposer une nouvelle accusation.

Devant ce type de résultats, certains concluent que l'appareil pénal n'est pas assez rigoureux face à la violence conjugale et demandent des taux de condamnation supérieurs. Face à une telle demande, deux remarques s'imposent. D'abord, le tribunal dispose de diverses mesures pour favoriser la dissuasion, qui ne sont pas du seul ressort de la sentence. L'arrêt des procédures, qui ouvre la porte à une éventuelle reprise de la poursuite, peut constituer une source de dissuasion pour l'inculpé. De plus, le tribunal dispose d'une mesure visant à assurer un certain encadrement du justiciable sans pour autant donner lieu à un casier judiciaire (art. 810 C.cr.). Cette mesure prévoit une obligation de garder la paix ainsi que l'imposition de conditions raisonnables qui seraient jugées nécessaires à cette fin, permettant dans certaines circonstances de protéger la victime présumée. Malheureusement, nous n'avons pas eu accès aux données concernant cette mesure et nous ne pouvons donc en évaluer l'utilisation réelle dans les causes étudiées.

Ensuite, toute analyse du traitement judiciaire des événements impliquant des conjoints doit tenir compte du contexte entourant la judicia- 
risation de ces affaires, donc des conditions qui en régissent l'entrée dans l'appareil judiciaire. Nous pouvons penser que, puisque les policiers doivent porter des accusations dans les affaires de violence conjugale, ils filtrent moins les causes. En d'autres termes, ils rejettent moins de situations. Dans ces circonstances, les affaires se rendant au tribunal ne comportent pas toujours les éléments de preuve que l'on retrouve dans les autres affaires criminelles. Or, une reconnaissance de culpabilité implique que le procureur réussisse à établir la preuve " hors de tout doute raisonnable » qu'une infraction a été commise. Les affaires conjugales se trouvent soumises aux mêmes règles de droit que toute autre affaire pénale et les accusés jouissent des mêmes protections juridiques. De plus, puisque dans les situations de violence conjugale la victime est très souvent le seul témoin de l'événement, la preuve risque de reposer sur ses seules épaules. Le taux élevé d'attrition ${ }^{11}$ des causes de violence conjugale s'explique en partie par le refus des victimes de collaborer aux procédures judiciaires. S'il nous est impossible de quantifier cette situation, il reste que la littérature fournit de nombreuses raisons qui peuvent expliquer ce genre de décision de la part de la victime.

La peur des représailles de la part du conjoint est souvent mentionnée comme étant le facteur le plus important pour expliquer le manque de coopération des victimes dans le processus judiciaire (Wangberg, 1991; Hart, 1993; Erez et Belknap, 1998; Bennett et al., 1999). Une victime peut également demander que la plainte soit retirée parce qu'elle a pardonné à l'agresseur, qu'elle s'est réconciliée avec lui ou qu'elle en est dépendante émotivement ou socio-économiquement (Buzawa et Buzawa, 1990; Wangberg, 1991; Corsilles, 1994; McGuire, 1997; Erez et Belknap, 1998). On dit aussi qu'une victime peut souhaiter l'interruption des procédures parce qu'elle vit la période de la lune de miel, dernière phase du cycle de la violence, au cours de laquelle le conjoint regrette son geste, devient doux, attentionné et promet qu'il ne recommencera pas (Morier et al., 1991). Ou encore que ce refus est une des manifestations du syndrome de la femme battue : la victime n'a plus confiance en elle et a perdu espoir que la situation change, ou alors elle se trouve dans un état d'impuissance acquise, i.e. qu'étant maltraitée depuis une longue période, la victime devient « insensibilisée » à la

11. Il faudra éventuellement distinguer entre les différentes formes juridiques menant à l'abandon temporaire ou définitif des procédures et leurs possibles effets sur les justiciables et les victimes. 
violence et est de moins en moins capable de se défendre et de se protéger (Law Reform Commission of Nova Scotia, 1995).

Plusieurs de ces explications, liées à la victime, sont parfois mises de l'avant pour justifier l'idée selon laquelle les intervenants pénaux sont mieux placés que les victimes pour déterminer la pertinence ou non d'une poursuite et des diverses décisions qui seront prises au cours des procédures, incluant celles sur la remise en liberté provisoire et la peine. Ce que les auteurs disent moins souvent, nous semble-t-il, c'est que certaines de ces femmes peuvent souhaiter l'arrêt des procédures de leur plein gré, en toute connaissance de cause. Elles peuvent considérer que ce type d'intervention dans leur vie ne leur convient pas; ceci nous semble d'autant plus plausible lorsque les poursuites ont été intentées contre leur gré ou qu'elles ont l'impression de ne pas avoir d'autre pouvoir que celui de faire condamner leur conjoint.

Outre les raisons mentionnées plus haut, on attribue également l'attrition au fait que les victimes manquent d'informations concernant l'intervention judiciaire et qu'elles ne sont pas suffisamment soutenues au cours des procédures (Wangberg, 1991; Hart, 1993; McGuire, 1997; Bennett et al.; 1999). L'attitude ou les comportements des intervenants judiciaires (procureurs de la couronne, juge et avocats de la défense) envers les victimes de violence conjugale sont aussi régulièrement ciblés pour expliquer leur refus de collaborer aux procédures (Ford et Regoli, 1993; Hart, 1993; Corsilles, 1994; Cretney et Davis, 1997; Erez et Belknap, 1998). La victime peut aussi hésiter ou refuser de s'impliquer parce qu'elle n'a pas confiance dans l'efficacité de l'appareil judiciaire ou alors parce qu'elle y a vécu des expériences difficiles (Erez et Belknap, 1998). La lenteur des procédures, qui peuvent durer plusieurs mois, peut aussi expliquer l'attrition (Wangberg, 1991; Hart, 1993; McGuire, 1997; Bennett et al., 1999) et le stress qui y est associé peut restreindre le désir d'une victime de s'impliquer plus avant (Cretney et Davis, 1997; Erez et Belknap, 1998). Finalement, certains se fondent sur une approche coûts-bénéfices : si une victime considère que les conséquences négatives des procédures sont trop élevées comparativement aux conséquences positives pouvant en découler, elle risque de perdre tout intérêt face à la poursuite (Ford, 1991; Hart, 1993).

Pour éviter que la preuve repose entièrement sur la victime, plusieurs suggèrent que les policiers recueillent de meilleures preuves, que les médecins témoignent des blessures subies, etc. (Corsilles, 1994; Rondeau et al. 1998). Nous sommes d'accord avec de telles suggestions, mais en 
partie seulement. Nous sommes d'avis que peu importe la manière dont la preuve est faite, il est nécessaire de tenir compte de ce que la victime souhaite. Ceci nous semble d'autant plus incontournable que souvent, les victimes ont appelé la police non pas pour que des poursuites soient intentées mais plutôt pour être protégées.

Pour contrer le taux d'attrition des causes de violence conjugale, diverses stratégies sont utilisées pour amener les victimes à témoigner contre leur partenaire. Que l'accusé finisse par plaider coupable ou soit déclaré coupable par un juge ou un jury, peu importe. L'important semble être l'obtention d'une condamnation. Mais à qui profite-t-elle ? Elle peut satisfaire ceux qui parlent au nom des victimes, ceux qui décident à leur place quel est le meilleur moyen pour régler leurs problèmes, ceux qui voient dans ces répressions individuelles une solution de premier plan pour lutter contre la violence conjugale. Elle peut aussi correspondre au désir de certaines victimes qui souhaitent que l'agresseur soit puni pour ce qu'il lui a fait. Or, si l'objectif visé par la judiciarisation n'est pas la seule réprobation symbolique mais la punition d'individus identifiables, sa mise en œuvre semble bien imparfaite. Dans notre recherche, un conjoint sur trois est coupable au terme des procédures.

Une condamnation peut également servir à valider l'implication des intervenants pénaux dans les affaires de violence conjugale, à confirmer les attentes que plusieurs ont placé dans cet appareil. Le travail des intervenants pénaux, dans la recherche d'une condamnation, contribue à sauvegarder les représentations du public face à ce système, particulièrement en ce qui a trait à sa performance et sa capacité d'amener les causes jusqu'au bout. La condamnation prouve que les intervenants contrôlent la situation, qu'ils répondent au mandat qui leur a été confié. En ce sens, une condamnation peut être bénéfique pour l'appareil judiciaire lui-même, puisque le coupable est officiellement reconnu comme tel, qu'il en portera la marque dans un casier judiciaire et surtout, qu'il sera sanctionné. Mais au bout du compte, une cause qui se termine de cette manière est-elle un succès pour la victime ? Pour l'appareil judiciaire ? Pour ceux qui militent en faveur de la judiciarisation ? Sur le plan symbolique aussi bien que matériel, le système pénal constitue l'ultime recours mais aussi la source des plus sévères sanctions disponibles dans nos sociétés. Pour les groupes de pression et pour le grand public, sans doute, la réprobation sociale de la violence conjugale doit se traduire par une condamnation et une peine sévère, à la hauteur des 
conséquences des actes violents. Nous venons de voir ce qu'il en était du taux de condamnation. Qu'en est-il de la sévérité de la peine ?

\section{La peine}

C'est au procureur de la couronne de faire valoir quel type de sentence le juge devrait imposer à l'accusé coupable. Pour ce faire, il évalue la gravité objective et subjective de l'infraction, la nécessité de protéger la victime et sa famille ainsi que les intérêts de la justice et de la société. Il choisira, d'après son évaluation, de recommander une sentence curative (réhabilitative), punitive ou ayant les deux composantes.

La question du type de sentence dont devraient écoper les hommes accusés de violence conjugale fait l'objet de beaucoup de débats. La sentence à privilégier pour les conjoints doit viser, entre autres, la cessation de la violence et la protection des victimes. La sentence imposée doit être dissuasive et punitive (Québec, 1998). L'amende n'est pas perçue comme une peine adéquate, puisqu'elle prive la famille d'un revenu. Ce n'est donc pas seulement l'accusé qui subit les conséquences de cette sentence. Quant à la prison, elle est susceptible de victimiser doublement les conjointes : cette peine peut ne pas correspondre à ce que la victime désire et les conséquences financières d'un emprisonnement sont souvent importantes, particulièrement lorsqu'elle entraîne la perte de l'emploi du conjoint (MacLeod, 1980; ONU, 1989). Certains réclament que les conjoints coupables écopent toujours d'une sentence de prison sévère, peu importe la nature de l'affaire, afin de démontrer que ces comportements sont inacceptables (Dwana Ring, membre dissidente de la Law Reform Commission of Nova Scotia, 1995). Pour d'autres, cette peine devrait être réservée aux cas graves (Gravel, 1993; Québec, 1998). D'autres encore défendent l'idée que la prison n'aurait pas les effets dissuasifs et curatifs qu'on lui prête (Robert, 1984; SaintLaurent, 1986; Landreville et Laberge, 1994). On craint aussi que la prison contribue à accroître la violence du conjoint (Soler, 1987; ONU, 1989; Law Reform Commission of Nova Scotia, 1995).

Nos données révèlent que parmi ceux coupables à l'issue des procédures, le tiers des conjoints $(36,8 \%)$ a reçu une sentence carcérale (tableau 2). La probation avec ou sans amende, sans qu'elle soit précédée d'un emprisonnement, est la peine la plus souvent imposée $(60,3 \%$ des cas). Les conjoints condamnés à la prison se voient imposer des peines plutôt courtes ( $84 \%$ sont condamnés à des peines d'emprisonnement de moins de trois mois). La situation est très différente pour les autres 
TABLEA U 2

La sentence

\begin{tabular}{|l|c|c|c|c|}
\hline & \multicolumn{2}{|c|}{ Violence conjugale } & \multicolumn{2}{c|}{$\begin{array}{c}\text { Autres infractions } \\
\text { contre la personne }\end{array}$} \\
\hline Sentence & N & $\%$ & N & $\%$ \\
\hline Type de sentence & & & & \\
amende & 2 & 2,9 & 2 & 1,3 \\
probation & 30 & 44,1 & 36 & 15,1 \\
probation et amende & 11 & 16,2 & 19 & 8,0 \\
emprisonnement & 5 & 7,4 & 97 & 40,8 \\
emprisonnement et probation & 20 & 29,4 & 83 & 34,9 \\
Total & 68 & 100 & 238 & 100 \\
\hline Durée d'emprisonnement ${ }^{2}$ & 10 & 40,0 & 14 & 7,8 \\
1 à 14 jours & 11 & 44,0 & 51 & 28,3 \\
15 jours à 3 mois & 4 & 16,0 & 57 & 31,7 \\
plus de 3 mois à 2 ans et moins & 0 & 0,0 & 58 & 32,2 \\
2 ans et plus & 25 & 100 & 180 & 100 \\
\hline Total & & & & \\
\hline
\end{tabular}

${ }^{1} \mathrm{Khi}^{2}: 43,31 ; \mathrm{p}: 0,00$; V de Cramer : 0,38. Valeurs présentes : 93,6\%.

${ }^{2} \mathrm{Khi}^{2}$ : 31,11; p : 0,00; V de Cramer : 0,39. Valeurs présentes : $100 \%$.

hommes : $75 \%$ d'entre eux ont reçu une sentence carcérale, 23,1\% ont reçu une sentence de probation (avec ou sans amende) et la durée des peines infligées est plus longue.

Le faible taux d'incarcération des conjoints coupables ou l'apparente clémence des peines prouve, selon certains, que les juges ne prennent pas la violence conjugale au sérieux ou que les conjoints bénéficient d'un traitement préférentiel à l'étape de la sentence (ONU, 1989; Brown, 1991; Côté, 1991; Dwana Ring, membre dissidente de la Law Reform Commission of Nova Scotia, 1995). Selon d'autres, les juges ne donnent pas souvent de sentences d'incarcération parce qu'ils tiennent compte des désirs des victimes, qui ne veulent pas nécessairement que leur conjoint soit emprisonné mais plutôt qu'il cesse d'être violent (MacLeod, 1980; Law Reform Commission of Nova Scotia, 1995). C'est pourquoi ils imposent des peines alternatives à l'incarcération, qui visent davantage la réhabilitation que la punition (Morier et al., 1991). C'est d'ailleurs ce que certains auteurs recommandent, sans nécessairement 
prétendre qu'aucun conjoint ne devrait être emprisonné (MacLeod, 1980; ASRSQ 1989) d'autant plus que la prison n'est qu'une mesure temporaire de neutralisation (ONU, 1989). Il est également possible que les juges, tenant compte des réserves émises à propos de la valeur réhabilitative et dissuasive de la prison, optent pour d'autres types de peine. Certes, une peine autre que l'emprisonnement entraîne moins de coûts personnels et sociaux qu'une peine carcérale. En ce sens, au plan des conséquences, ceux qui évitent cette issue sont potentiellement moins pénalisés que les autres. Par contre, les autres peines ne doivent pas être conçues comme un traitement de faveur. Si les coûts qu'elles entraînent sont moindres, elles ne sont pas pour autant sans conséquences ou sans contraintes.

La thérapie est une des options qui s'offrent au juge au cours du processus judiciaire. À ce sujet, deux remarques s'imposent. En premier lieu, certaines ressources pour conjoints violents refusent d'intervenir auprès d'eux tant qu'ils n'ont pas fait l'objet d'une sentence. Or, la question du momentum est importante : toute intervention psychosociale devrait être amorcée le plus tôt possible. Dans la mesure où l'arrestation cause un choc à certains d'entre eux, ce moment est peut-être un des plus appropriés pour amorcer une réflexion sur les conséquences des comportements violents et sur les démarches à entreprendre pour qu'ils ne se reproduisent plus. En second lieu, certains arguent qu'on ne peut pas forcer un conjoint violent à aller en thérapie, qu'il doit entreprendre volontairement ce processus pour que cela ait un effet. D'autres affirment qu'un conjoint est rarement totalement volontaire pour entreprendre cette démarche, qu'il le fera suite aux pressions exercées par sa partenaire ou parce qu'il a peur de la perdre. En quoi la menace d'entreprendre des procédures judiciaires serait-elle moins efficace que les autres types de motivation? Pourquoi ne pas tenter quand même l'intervention ? Pourquoi l'accusé devrait-il d'abord admettre qu'il a un problème avant que les ressources acceptent de le prendre en charge ? Pourquoi des tiers ne pourraient-ils pas aider ces hommes à prendre conscience qu'ils se comportent de manière inacceptable ? Évidemment, les ressources étant peu nombreuses et pouvant connaître des problèmes de financement, il est compréhensible que les intervenants ne souhaitent travailler qu'avec les hommes qui sont volontaires pour entreprendre cette démarche. Mais si plus de places dans ces ressources étaient disponibles, auraient-ils les mêmes réticences? 


\section{Conclusion}

Nous avons vu que les causes de violence conjugale à l'étude ne se terminent pas en majorité par une culpabilité. Nous avons également constaté que dans le tiers des cas, les accusés coupables sont condamnés à la prison. Ces résultats sont-ils le signe d'une sous-performance du système ? Nous ne le croyons pas et nous avons offert plusieurs types d'interprétation pour expliquer ces situations. Néanmoins, des recherches seront indispensables pour éclaircir certains points : comment peuton mieux expliquer les diverses décisions qui font en sorte que les causes ne se terminent pas par une culpabilité ou un acquittement? pour quelles raisons et à quelle étape les accusés plaident-ils coupables ? dans quelle mesure et dans quelles circonstances les dispositions prévues à l'article 810 du code criminel sont-elles utilisées ? à quelle fréquence les conjoints accusés sont-ils renvoyés en thérapie à l'une ou l'autre des étapes judiciaires?

Nombreux sont ceux qui admettent que la judiciarisation ou l'emprisonnement ne sont pas la solution qui réglera le problème sérieux que constitue la violence conjugale. Aucune solution unique, pas même les thérapies pour conjoints violents, ne peut avoir cette prétention. L'intervention pénale a été mise de l'avant avec une énorme pression sociale et elle est apparue pour plusieurs comme la solution incontournable pour gérer les affaires de violence conjugale. Malheureusement, il semble que les énergies des dernières années aient été consacrées à augmenter l'efficacité de la gestion pénale des affaires de violence conjugale plutôt qu'à développer un éventail diversifié de stratégies. À ce sujet, il faut souligner que la réhabilitation n'est pas exclusivement le résultat de l'intervention pénale comme telle, mais plutôt le résultat attendu des interventions effectuées dans le cadre de certains programmes intra ou extra judiciaires. On peut d'ailleurs craindre que les situations ayant des effets positifs aillent en diminuant dans un contexte de coupures budgétaires et de réduction des services. Contentons-nous de souligner la quasi-absence de programmes de thérapie pour conjoints violents dans les établissements de détention provinciaux ${ }^{12}$ (Lemire et al., 1996).

Une politique de prévention et de lutte contre la violence conjugale en accord avec ses prétentions devrait faire en sorte de répondre aux attentes qu'elle suscite et assurer la disponibilité des mesures qui visent la réhabilitation des conjoints violents. Il nous semble pourtant que la

12. Où sont détenus ceux condamnés à des peines d'incarcération de deux ans et moins. 
logique pénale prend le pas sur toute autre perspective. La confiance placée dans la capacité de l'appareil pénal de transformer des individus mais surtout la qualification de toute manifestation de violence conjugale comme crime font en sorte qu'il peut être difficile de concevoir des alternatives à sa judiciarisation. N'y aurait-il pas lieu d'imaginer d'autres options dans certains cas ? Il ne faut pas confondre «alternative » avec « absence de contrôle ». Pour plusieurs, parler d'alternative à la judiciarisation équivaut à dépénaliser un comportement. Il n'en est rien. Nous connaissons le contexte dans lequel les groupes de pression ont réussi à imposer une représentation de la violence conjugale en tant que crime. La sensibilisation à cette problématique n'est pas terminée. Par contre, on ne peut pas prétendre que l'intervention judiciaire soit une panacée, qu'elle soit le seul mode de contrôle social efficace pour protéger les victimes et réhabiliter les agresseurs.

Nous ne nous opposons pas à l'usage de la menace de poursuites pénales comme forme de contrainte. Avec l'aide des experts intervenant auprès des femmes et des hommes aux prises avec ce problème, avec l'implication de professionnels œuvrant dans d'autres milieux, incluant les intervenants pénaux, il y a peut-être moyen d'en arriver à d'autres solutions qui seraient plus efficaces en termes de prévention de l'agir violent ou de la récidive. Il est important d'éviter que des mesures soient prises pour accroître la performance du système si ces mesures ne sont pas nécessaires ou encore si elles ont pour effet d'alourdir la situation du couple ou pire, d'exposer la victime elle-même à des mesures judiciaires.

Nous proposons que, dans certaines situations, le contrôle pénal soit remplacé par un autre type de contrôle, avec l'accord de la victime et sa participation, si elle le souhaite. Rappelons que la réprobation sociale ne peut être réduite à la seule répression pénale mais qu'elle peut prendre la forme d'un contrôle institutionnel ou communautaire. Évidemment, il est nécessaire d'évaluer chaque situation avant de mettre en ouvre ces réponses. Cette évaluation pourrait s'effectuer dès après l'intervention policière. Lorsque les policiers sont appelés sur les lieux d'une affaire de violence conjugale, nous suggérons, tel que cela est fait actuellement, que l'accusé soit arrêté et amené au poste de police. Par contre, au lieu de procéder de la manière habituelle, i.e. de déposer des accusations en présence d'une preuve suffisante, nous proposons l'enclenchement d'une procédure d'évaluation par une équipe d'intervenants spécialisés. Cette évaluation pourrait être reportée de quelques heures, particulièrement lorsque la victime est trop ébranlée pour y participer. Si la victime est 
d'accord et qu' elle se sent prête à le faire, une intervenante œuvrant auprès des femmes victimes de violence pourrait se rendre chez elle, lui expliquant d'emblée les motifs de sa présence. Une victime qui vient de vivre l'arrestation de son partenaire ou de son ex-conjoint, suite à une agression ou à des menaces, a sans aucun doute besoin de soutien et de réconfort, de parler de ce qui vient d'arriver et des expériences vécues antérieurement. L'intervenante pourrait ensuite évaluer son niveau d'inquiétude et lui présenter les options qui s'offrent à elle et à son partenaire ${ }^{13}$, en la consultant à ce sujet. Ces intervenants, après rencontre avec la victime chez elle et avec l'accusé au poste, détermineraient s'il y a lieu de judiciariser ou non la situation. La judiciarisation pourrait être, entre autres, réservée aux situations d'infractions graves, à ceux qui refusent de se soumettre aux mesures proposées ou qui persistent à se représenter comme une victime dans l'affaire. Ceux-là suivront la filière la plus punitive. Là pourrait se situer la manifestation la plus extrême et la plus lourde de conséquence de la réprobation sociale.

\section{Références}

Amoretti, A., Cousineau, M.-M., Gauthier, S., Laberge, D., Landreville, P., Michaud, L., Mordente, S., ThéOrêt, B. et Trkulja, J. 1996. La détention sous garde et la mise en liberté provisoire à la Cour du Québec, à Montréal, Rapport final, Montréal : Groupe de recherche et d'analyse sur les politiques et les pratiques pénales.

Association des services de réhabilitation sociale du Québec 1989. La violence conjugale au Québec. L'intervention auprès des conjoints violents par une approche communautaire concertée, Montréal : ASRSQ.

Beaudry, M. 1984. Les Maisons des femmes battues au Québec, Montréal : SaintMartin.

Bennett, L., Goodman, L. et Dutton, M.A. 1999. «Systemic Obstacles to the Criminal Prosecution of a Battering Women » Journal of Interpersonal Violence 14 (7) : 761-772.

British Columbia Task Force on Family Violence. 1992. Is Anyone Listening? Report on the British Columbia Task Force on Family Violence, Victoria, C.B. : Ministry of Women's Equality.

Brodeur, J.-P. et LANDreville, P. 1979. Finalités du système de l'administration de la justice pénale et planification des politiques, Les cahiers de l'École de criminologie, cahier no 2, Montréal : Université de Montréal.

13. Par exemple, thérapie individuelle ou de couple, médiation, divorce, sessions de sensibilisation à la violence. 
Brown, M.G. (Ed.) 1991. Gender Equality in the Courts Criminal Law: A Study by the Manitoba Association of Women and the Law, Manitoba : Victims Assistance Committee.

Burris, C.A. et JAfFe, P. 1983. «Wife Abuse as a Crime: The Impact of Police Laying Charges » Canadian Journal of Criminology 25 : 309-318.

BusCH, R. et Robertson, N.R. 1993. «What's Love Got to Do With It ? An Analysis of an Intervention Approach to Domestic Violence » Waikato Law Review 1 : 109-140.

Busch, R., Robertson, N.R. et LAPSLEy, H. 1992. Protection from Family Violence. A Study of Protection Orders Under the Domestic Protection Act 1982 (Abridged), Hamilton, NZ : University of Waikato.

Buzawa, E.S. et Buzawa, C.G. 1990. Domestic Violence: The Criminal Justice Response. Studies in Crime, Law and Justice (vol. 6), Newbury Park : Sage.

Canada. Division de la prévention de la violence familiale 1991. Violence familiale : document sur la situation, Ottawa : Gouvernement du Canada.

Commission de réforme du droit du Canada 1976. Notre droit pénal (Rapport no 3), Ottawa : Commission de réforme du droit du Canada.

Corsilles, A. 1994. "No-Drop Policies in the Prosecution of Domestic Violence Cases: Guarantee to Action or Dangerous Solution ? » Fordham Law Review LXIII (3) : 853-881.

CôTÉ, L. 1991. Les tribunaux et la violence conjugale : le portrait dans deux districts judiciaires, Montréal : Regroupement provincial des maisons d'hébergement et de transition pour femmes victimes de violence conjugale.

Cretney, A. et Davis, G. 1997. «Prosecuting Domestic Assault: Victims Failing Courts, or Courts Failing Victims ? » The Howard Journal 36 (2) : 146-157.

DAVIS, N.J. 1988. «Battered Women: Implications for Social Control» Contemporary Crises 12 (4) : 345-372.

DEMERS, C. 1990. Étude descriptive du traitement de la violence conjugale par le programme de conciliation de la Cour municipale de Montréal, Les cahiers du GRAPPP, cahier no 9, Montréal : Université de Montréal, Université du Québec à Montréal.

EREZ, E. et BelKnAP, J. 1998. «In Their Own Words: Battered Women's Assessment of the Criminal Processing System's Responses »Violence and Victims 13 (3) : 251-268.

ERICSON, R.V. et BARANEK, P.M. 1982. The Ordering of Justice: A Study of Accused Persons as Dependants in the Criminal Process, Toronto : University of Toronto Press.

FORD, D.A. 1991. "Prosecution as a Victim Power Resource: A Note on Empowering Women in Violent Conjugal Relationships »Law $\mathcal{V}$ Society Review 25 (2) : 313-334.

ForD, D.A. et Regoli, M.J. 1993. "The Criminal Prosecution of Wife Assaulters: Process, Problems, and Effects », Pp. 127-164 in Legal Responses to Wife Assault: Current Trends and Evaluation, sous la direction de Z.N. Hilton. Thousand Oaks : Sage Publications.

GAUTHIER, S. 1998. Le traitement judiciaire de la violence conjugale : analyse comparée d'une cohorte de justiciables, Thèse présentée comme exigence partielle du doctorat en sociologie. Montréal : Université du Québec à Montréal. 
Gelles, R.J. et Straus, M.A. 1988. Intimate Violence: The Causes and Consequences of Abuse in the American Family, New York : Simon \& Schuster.

Gondolf, E.W. et MCFerRoN, R.J. 1989. «Handling Battering Men: Police Action in Wife Abuse Cases » Criminal Justice and Behavior 16 (4) : 429-439.

Gravel, E. 1993. «La nécessité d'un traitement judiciaire », Pp. 145-147 in Violence et déviance à Montréal, sous la direction de M. Chalom et J. Kousik. Montréal : Liber.

HART, B.J. 1993. « Battered Women and the Criminal Justice System » American Behavioral Scientist 36 (5) : 624-638.

JONES, A. 1994. Next Time, She'll be Dead: Battering and How to Stop it, Boston : Beacon Press.

LABERGE, D. et LANDREVILle, P. 1994. "La judiciarisation des problèmes sociaux », Pp. 1053-1066 in Traité des problèmes sociaux., sous la direction de F. Dumont, S. Langlois et Y. Martin. Québec : Institut québécois de recherche sur la culture.

Landreville, P., Blankevoort, V. et Pires, A.P. 1981. Les coûts sociaux du système pénal, Montréal : Université de Montréal.

LANDREVILle, P. et LABERGE, D. 1994. «La prison, solution au problème social ? », Pp. 1067-1080 in Traité des problèmes sociaux, sous la direction de F. Dumont, S. Langlois et Y. Martin. Québec : Institut québécois de recherche sur la culture.

LAVERGNE, C. 1997. Analyse du processus de construction de la violence faite aux femmes en milieu conjugal comme problème socio-pénal au Québec, Thèse présentée à la faculté des études supérieures en vue de l'obtention du grade de Ph.D., Montréal : Université de Montréal.

Law Reform Commission of Nova Scotia. 1995. From Rhetoric to Reality: Ending Domestic Violence in Nova Scotia, Halifax : Law Reform Commission of Nova Scotia.

Lemire, G., Rondeau, G., Brochu, S., Schneeberger, P. et Brodeur, N. 1996. "Les programmes de traitement pour hommes violents: du communautaire au correctionnel » Revue canadienne de criminologie (1) : 33-59.

MACLEOD, L. 1980. La femme battue au Canada : un cercle vicieux, Ottawa : Conseil consultatif canadien de la situation de la femme.

MaCLEOD, L. 1987. Pour de vraies amours... Prévenir la violence conjugale, Ottawa : Conseil consultatif canadien sur la situation de la femme.

MACLEOD, L. 1989. La violence conjugale : comprendre pour prévenir (rapport sur la séance de travail), Ottawa : Conseil consultatif canadien sur la situation de la femme.

MACLEOD, L. et PICARD, C. 1989. Pour une intervention plus efficace du système de justice pénale en matière de violence faite aux femmes : étude des limites et du potentiel d'une intervention efficace (document de travail), Ottawa : Ministère de la Justice.

MARTIN, D. 1987. « The Historical Roots of Domestic Violence », Pp. 3-20 in Domestic Violence on Trial: Psychological and Legal Dimensions of Family Violence, sous la direction de D.J. Sonkin. New York : Springer Publishing Company.

MCGUiRE, L.A. Novembre 1997. « Criminal Prosecution of Domestic Violence » Document Internet, for Battered Women's Justice Project, Récupéré le 20 juin 1999 du site www: http://www.vaw.umn.edu/BWJP/prosecutev.htm. 
Morgan, P. 1981. "From Battered Wife to Program Client: The State's Shaping of Social Problems » Kapitalistate $9:$ 17-39.

Morier, Y., Bluteau, C., Bruneau, G., Lessard, C. et Beaudet, P. 1991. Intervention socio-judiciaire en violence conjugale, Montréal : Wilson \& Lafleur Ltée.

Office des Nations Unies. Centre pour le développement social et les affaires humanitaires 1989. La violence contre les femmes dans la famille, New York: Nations Unies.

Québec 1986. Politique d'intervention en matière de violence conjugale, Québec : Ministère de la Justice. Ministère du Solliciteur général.

Québec 1995. Politique d'intervention en matière de violence conjugale: prévenir, dépister et contrer la violence conjugale, Québec : Ministère de la Santé et des Services sociaux. Ministère de la Justice. Secrétariat à la condition féminine. Ministère de la Sécurité publique. Ministère de l'Éducation. Secrétariat à la famille.

Québec 1998. Le substitut du procureur général et la violence conjugale, Québec : Ministère de la Justice. Récupéré le 2 mai 1999 du site WWW : http:// www.justice.gouv.qc.ca/francais/ publication/public/substit.htm

ROBERT, P. 1984. La question pénale, Genève : Droz.

Rondeau, G., Castonguay, S. Brochu, S. et Fredette, C. 1998. "L'utilisation du système pénal dans les situations de violence conjugale au Québec (rapport no 3) », Pp. 81-145 in Le recours au droit pénal et au système pénal pour régler les problèmes sociaux, sous la direction de G. Lemire, P. Noreau, G. Rondeau, S. Castonguay, S. Brochu, J. Proulx, C. Langlois, et C. Fredette. Montréal : CICC, Université de Montréal.

Saint-Laurent, D. 1986. Principes de droit constitutionnel et pénal, Mont-Royal : Modulo.

SOler, E. 1987. « Domestic Violence Is a Crime : A Case Study - San Francisco Family Violence Project», Pp. 21-35 in Domestic Violence on Trial: Psychological and Legal Dimensions of Family Violence, sous la direction de D.J. Sonkin. New York : Springer Publishing Company.

Statistique Canada. Centre canadien de la statistique juridique. 1994. La violence familiale au Canada : Canada, les données nationales et actuelles, Ottawa : Ministre de l'Industrie, des Sciences et de la Technologie.

URSEL, E.J. 1994. « Le tribunal de la violence familiale à Winnipeg » Juristat 14 (12) : $1-14$

WALKER, G.A. 1990. Family Violence and the Women's Movement: The Conceptual Politics of Struggle, Toronto : University of Toronto Press.

WangBerg, K.G. 1991. « Reducing Case Attrition in Domestic Violence Cases: A Prosecutor's Perspective » Prosecutor 24 (3) : 8-12. 